\title{
Calibration CAPABILITY WiTH LASER FREQUENCY STANDARD
}

\author{
LIPUS, L.C., BUDZYN, G., RZEPKA, J. \& ACKO, B.
}

Abstract: Highest-level traceability of dimensional measurements in the Laboratory for Production Measurement (LTM) at University of Maribor (a nationally designated metrology institute), consistent with the Mutual Recognition Arrangement by the International Committee for Weights and Measure, is presented. A reliable primary laser frequency standard is important for national chains of industrial calibrations and measurements. Laser stabilization technique and calibration procedure for investigating the stability and reproducibility of HeNe lasers, used for interferometry length measurements, are described. Along the international interlaboratory comparisons, the calibration accuracy is periodically verified using a highly stable interferometer laser, by a simple method presented in this contribution.

Key words: laser, frequency stability, calibration, traceability
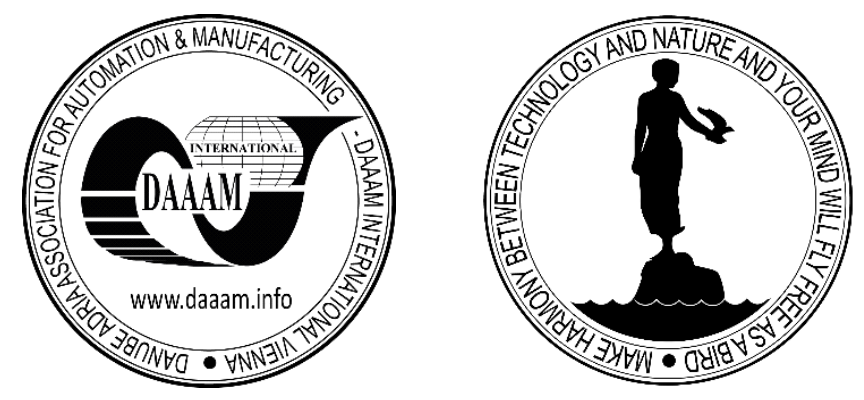

Authors' data: Univ. Prof. Dr. Sc. Acko, B[ojan] \& Assist. Dr. Sc. Lipus, C[repinsek] L[ucija], University of Maribor, Faculty of Mechanical Engineering, Smetanova 17, 2000 Maribor, Slovenia; Univ. Prof. Dr. Sc. Rzepka, J[anusz] \& Assist. Prof. Dr. Sc. Budzyn, G[rzegorz], Wroclaw University of Technology, Faculty of Electronics, Janiszewskiego 7, 50-370 Wroclaw, Poland; lucija.lipus@um.si, grzegorz.budzyn@pwr.wroc.pl, janusz.rzepka@pwr.wroc.pl, bojan.acko@um.si

This Publication has to be referred as: C. Lipus, L[ucija]; Budzyn, G[rzegorz]; Rzepka, J[anusz] \& Acko, B[ojan] (2016). Calibration Capability With Laser Capability Standard, Chapter 18 in DAAAM International Scientific Book 2016, pp.197-206, B. Katalinic (Ed.), Published by DAAAM International, ISBN 978-3902734-09-9, ISSN 1726-9687, Vienna, Austria

DOI:10.2507/daaam.scibook.2016.18 
C. Lipus, L.; Budzyn, G.; Rzepka, J. \& Acko, B.: Calibration Capability With Laser...

\section{Introduction}

The measurement system for the frequency/wavelength calibration of $\mathrm{HeNe}$ lasers used for industrial and laboratory interferometers and stability/reproducibility investigation of laser radiation was applied at LTM (as EURAMET national designated metrology institute, Faculty of Mechanical Engineering, University of Maribor).

At the time of adoption of the present definition of meter (as the length of the path traveled by light in vacuum during a time interval of 1/299 792458 of a second) by the $17^{\text {th }}$ General Conference (1983) CIPM (International Committee for Weights and Measures) drew up recommendations for the practical realization of the definition, called Mise en Pratique (MeP). The current MeP (Quinn, 2003), approved by CIPM (2002), recommends the Frequency of f-component $f_{\mathrm{f}}=473612353604$ $\mathrm{kHz}$ and the relative standard uncertainty of $2.1 \cdot 10^{-11}$. To ensure the stated standard uncertainty appropriate optical and electronic control systems are necessary.

The calibration system with standard laser (iodine-stabilized $\mathrm{HeNe}$ laser, named MIRS1), was developed by the Institute of Telecommunications and Acoustics, Wroclaw University of Technology (Budzyn et al, 2007; Budzyn \& Rzepka, 2008). LTM joined the CCL-K11 key comparison, the on-going intercomparison of primary standard lasers supported by the CIPM. According to the frequency stability of MIRS1, determined by comb measurements at the pilot laboratory Bundesamt für Eich- und Vermessungswesen, Austria (Matus et al, 2010), the relevant calibration and measurement capabilities were included into CIPM database.

Reliable primary frequency standard is of highest national metrological interest in the sense of assuring national traceability chains to high level industrial calibrations and measurements. At the highest accuracy level, the wavelength of a primary frequency standard is directly applied for calibrating mechanical standards such as gauge blocks (Mudronja et al, 2014). Primary frequency standard is the reference for frequency calibration of secondary HeNe lasers that are used in industry (Vorotnikov et al, 2016) and laboratory calibrations with high accuracy level (Godina \& Acko, 2012). Proving the national calibration and measurement capabilities through international interlaboratory comparisons and disseminating SI unit meter to accredited and industrial metrological laboratories through national interlaboratory comparisons are very important. LTM in the role of the EURAMET DI is steadily involved in international and regional intercomparison projects (Acko et al, 2013, 2014, 2015).

\section{Stabilization of radiation frequency}

In the $\mathrm{HeNe}$ gas mixture, helium atoms are excited to meta-stable states by electrons in an electrical discharge. At collisions with neon atoms, they transfer the excitation energy to neon, which radiates with a variety of different transitions into energetically lower states. The transition $3 \mathrm{~s}^{2} \rightarrow 2 \mathrm{p}^{4}$ radiates the red light with a frequency $v_{0}=474 \mathrm{THz}$ that is not a single frequency, but always has the frequency 
width (Reihle, 2004). The narrowest line-width is in a dilute gas at very low temperature; it broadens with increasing gas pressure (collision broadening) and with increasing temperature (Doppler broadening). For the light emitted from radiative $\mathrm{Ne}$ atom along the z-axis of the laser tube (which has a frequency of $v_{0}$ ) the frequency is broadened due to Doppler shift $v-v_{0}=v_{0} u_{\mathrm{z}} / c$ at light velocity $\mathrm{c}$. The velocity $u_{\mathrm{z}}$ is determined by the Maxwell-Boltzmann distribution (1), where $m$ is atom mass, $T$ is gas temperature and Boltzmann constant $k_{\mathrm{B}}=1.38 \cdot 10^{-23} \mathrm{~J} / \mathrm{K}$.

$$
F\left(u_{z}\right)=F_{0} \exp \left(-\frac{m u_{z}^{2}}{2 k_{B} T}\right)
$$

Inserting $u_{\mathrm{z}}$, the distribution is Gaussian function of $v$ :

$$
F(v)=F_{0} \exp \left(-\frac{m c^{2}\left(v-v_{0}\right)^{2}}{2 k_{B} T v_{0}^{2}}\right)
$$

The full width at half of maximum (FWHM, Fig. 1a) of (2) is

$$
\Delta v_{D}=2 v_{0} \sqrt{\frac{2 k_{B} T \ln 2}{m c^{2}}}
$$

For Ne atoms, taking $m \approx 3.3 \cdot 10^{-26} \mathrm{~kg}, T \approx 300 \mathrm{~K}$ and $v_{0} \approx 474 \mathrm{THz}$, the Doppler broadening is $\Delta v_{\mathrm{D}} \approx 1.3 \mathrm{GHz}$ (Fig. 1.b).

HeNe laser requires a long path through the active medium to obtain sufficient optical gain. This is achieved with multiple reflections between a pair of shaped mirrors with high reflections (Fabry-Perot resonator). The resonance condition at normal incidence is $L=N \lambda / 2$, where $L$ is the optical length between mirrors and $N$ is a large integer. So, the resonance frequencies are $v_{\mathrm{N}}=N c / 2 L$, and the frequency interval between successive resonance, known as the free spectral range (FSR) of the interferometer, is $\Delta v_{\mathrm{FSR}}=v_{\mathrm{N}+1^{-}}-v_{\mathrm{N}}=c / 2 L$ (Fig. 1.b). The resonance frequencies inside the Fabry-Perot resonator with flat mirrors have FWHM:

$$
\Delta v_{L}=\frac{c}{2 L} \cdot \frac{1-R_{1} R_{2}}{\pi \sqrt{R_{1} R_{2}}}
$$

Here the mirrors' reflectivity, $R_{1}$ and $R_{2}$, is very close to 1 . Practically, one of the mirrors is arranged to be as near as possible to $100 \%$ reflecting and the output mirror with a somewhat smaller reflectivity. For laser with $R_{1}=0.997$ and $R_{2}=0.994$ and the mirror distance $L$ varying from $139.02 \mathrm{~mm}$ to $139.28 \mathrm{~mm}$, we get $\Delta v_{L} \approx$ (3.091 to 3.097) $\mathrm{MHz}$ and $\Delta v_{\mathrm{FSR}} \approx 1.1 \mathrm{GHz}$, which means one resonant peak inside the FWHM of Doppler broadening (i.e. one $\Delta v_{\mathrm{L}}$ inside the range $\Delta v_{\mathrm{D}}$, Fig. 1.b). 

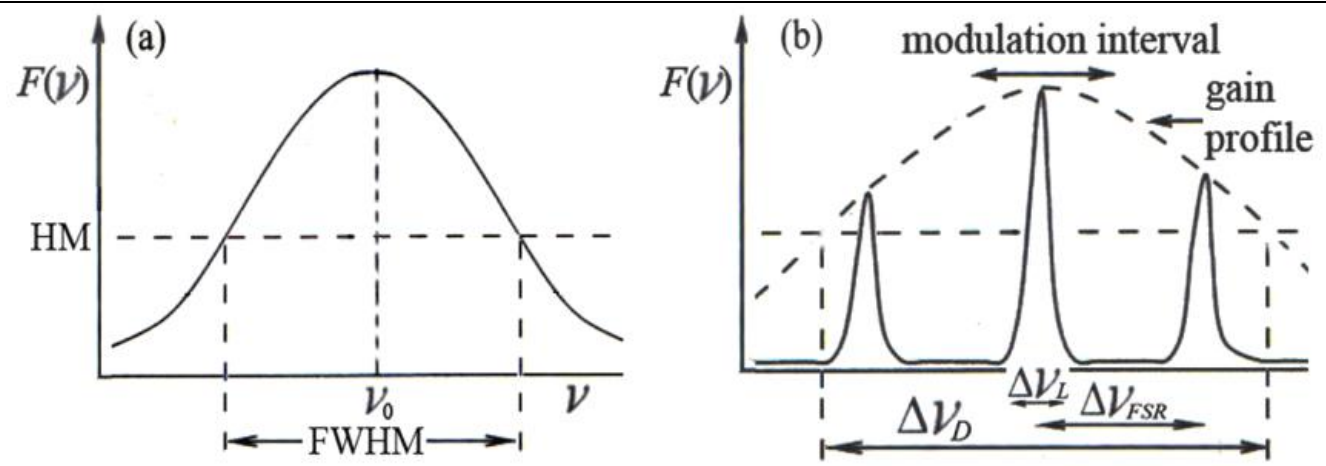

Fig. 1. Radiation-power distribution in Fabry-Perot resonator: (a) Full width at half maximum of Gaussian gain profile, (b) Three resonant peaks inside the gain profile

For stabilization of the resonant peak, an absorber cell containing molecular iodine vapor is inserted into the laser tube. The saturated absorption peak (with maximum at $v_{0}$ ) at has distribution of radiation power as Lorentzian function (where $2 \alpha$ is FWHM):

$$
F(v)=\frac{\alpha^{2}}{\alpha^{2}+\left(v-v_{0}\right)^{2}}
$$

For locking an iodine peak, the search begins with shifting the resonant peak along the top of the gain profile (in the interval of a few-hundred $\mathrm{MHz}$ ) by changing the cavity length as the mirror is precisely shifted by the piezoelectric transducer. The intensity of the resonant peak follows the Gaussian gain profile curve, but when crossing an iodine peak, it varies (it gets higher because of saturated absorption with Lorentzian term). The electronic servo system for tuning the laser frequency is based on the third derivation of the radiation power function. The Gaussian term is very broad and can be approximated in small intervals as a quadratic background, so the third derivation is the most appropriate, as this background is eliminated and the maximum of the Lorentzian term can be well determined. Third derivation of (5) gives (6), from which zero-crossing $v_{0}$ can be precisely defined between the symmetrical zero-crossings $v_{0} \pm \alpha$ (Fig. 2).

$$
F^{\prime \prime \prime}(v)=\frac{24 \alpha^{2}}{\left(\alpha^{2}+\left(v-v_{0}\right)^{2}\right)^{4}}\left(v-v_{0}\right)\left(\alpha^{2}-\left(v-v_{0}\right)^{2}\right)
$$

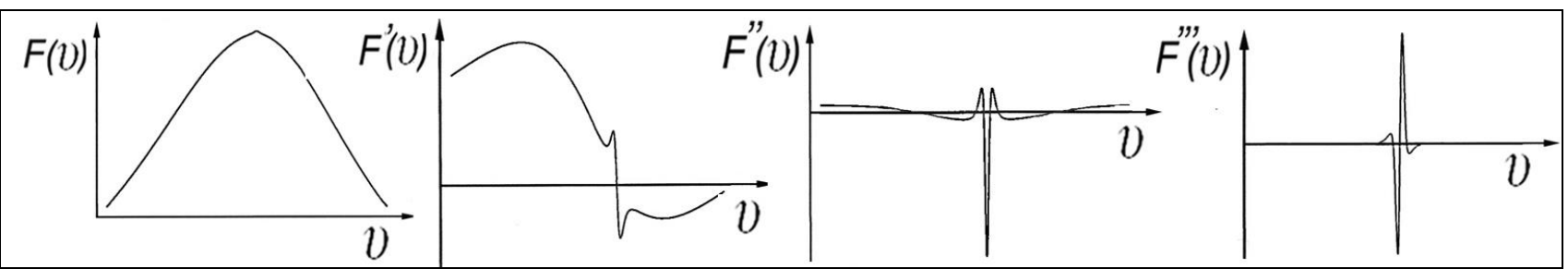

Fig. 2. The first, second and the third derivation of the power distribution 
For tuning the frequency, the mirror position is modulated with the frequency $v_{\mathrm{m}}=$ $1.6 \mathrm{kHz}$ and the amplitude $A_{m}=6.0 \pm 0.3 \mathrm{MHz}$, varying the output power with

$$
I=F\left(v+A_{m} \cos \omega t\right) ; \omega=2 \pi v_{m}
$$

For small $A_{m}$, it can be expressed by the Taylor series (8) and then according to the trigonometric rules for harmonic functions as (9).

$$
\begin{aligned}
& I=F(v)+\sum_{k=1}^{\infty}\left[\frac{\left(A_{m} \cos \omega t\right)^{k}}{k !} F^{(k)}(v)\right] \\
& I=C_{0}+C_{1} \cos \omega t+C_{2} \cos 2 \omega t+C_{3} \cos 3 \omega t+\ldots ; \\
& C_{0}=F(v)+\frac{A_{m}^{2} F^{\prime \prime}(v)}{4}+\ldots ; \quad C_{1}=A_{m} F^{\prime}(v)+\frac{A_{m}^{3} F^{\prime \prime \prime}(v)}{8}+\ldots ; \\
& C_{2}=\frac{A_{m}^{2}}{4} F^{\prime \prime}(v)+\frac{A_{m}^{4}}{48} F^{(4)}(v)+\ldots ; \quad C_{3}=\frac{A_{m}^{3}}{24} F^{\prime \prime \prime}(v)+\frac{A_{m}^{5}}{384} F^{(5)}(v)+\ldots
\end{aligned}
$$

The basic frequency $\omega$ is determined by third harmonic technique, where the function $C_{3} \cos 3 \omega t$ is filtered from the function (9), using the high-Q bandpass filtered detector. The first term in $C_{3}$ is much greater than the sum of all following terms, thus the signal filtered from (9) is proportional to $F^{\prime \prime \prime}(v)$ by $(6)$.

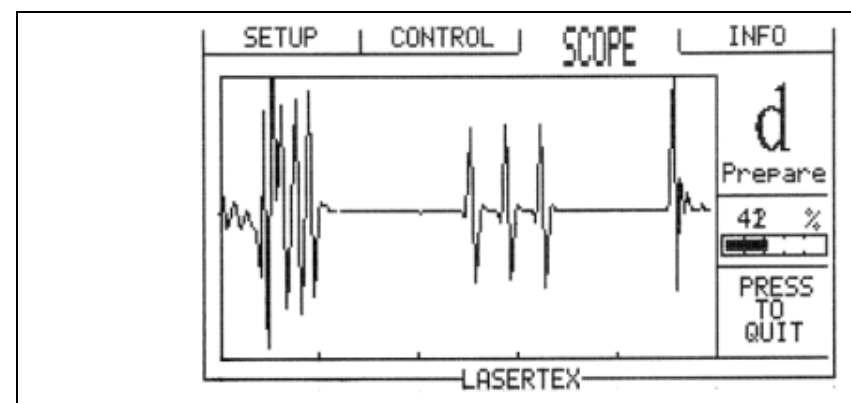

(a) Triplet of h, i, j peaks

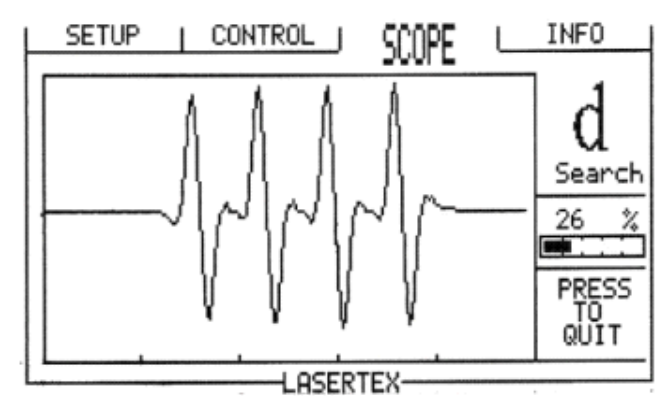

(b) Quadruplet of $d, e, f$, g peaks

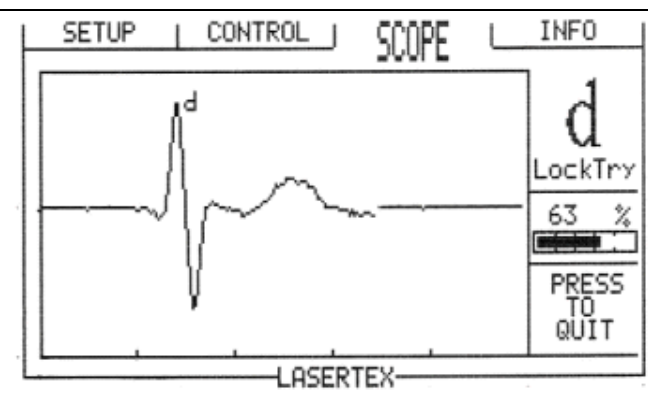

(c) Locking the peakd

\begin{tabular}{|c|c|}
\hline peak & $v_{1}(\mathrm{MHz})$ \\
\hline j & $473612 \mathbf{1 9 3 . 1 4 7}$ \\
\hline i & $473612 \mathbf{2 1 4 . 7 1 2}$ \\
\hline h & $473612 \mathbf{2 3 6 . 6 5 1}$ \\
\hline g & $473612 \mathbf{3 4 0 . 4 0 6}$ \\
\hline f & $473612 \mathbf{3 5 3 . 6 0 4}$ \\
\hline e & $473612 \mathbf{3 6 6 . 9 6 7}$ \\
\hline d & $473612 \mathbf{3 7 9 . 8 2 8}$ \\
\hline
\end{tabular}

(d) Peak's frequencies

Fig. 3. A signal displayed on the controller box during locking a chosen peak d:

(a) during mirrors' distance is increasing; (b) after automatic resetting of mirrors' distance onto starting position; (c) locking the peak; (d) Frequency values of peaks 
The resonator is made of invar tube located between the two mirrors, both attached to piezoelectric transducer actuators, so the cavity length can be adjusted by a voltage supply. The operational principle is to keep the laser wavelength resonant to one of hyper-fine transition of molecular iodine (Fig. 3.d), controlled by the third harmonic technique (signal in Fig. 3.c).

The control electronics of the laser enables the adjustment of the control parameters to the changing characteristic of the laser and the control of the device during operation. Starting the laser, the temperature of the internal resonator is stabilized within two hours. The user can observe the hyperfine components displayed, and lock the chosen frequency $v_{1}$ (Fig. 3.a/b). In normal laboratory conditions the laser is able to keep peaks locked for weeks.

Factors that can influence the determination of the correct center frequency of a chosen peak are iodine cell vapor pressure, modulation amplitude, intracavity laser power and the temperature of the wall of the iodine cell, and electronic offsets. The parameters for MIRS1, used in calculations of frequency widths, are adjusted to the values recommended by CIPM (Quinn, 2003, Reihle, 2004).

\section{Calibration of secondary laser}

The primary laser standard is commonly used in many laboratories around the world as the primary reference for calibrating the frequency of secondary lasers, which are employed in industry from large-coordinate to micro-system measurements.

The calibration system (Fig. 4) is applied for the definition radiation frequency/wavelength and investigation of frequency stability and reproducibility of $\mathrm{HeNe}$ laser interferometers (with stable red light of nominal vacuum wavelength in the range from $632.990 \mathrm{~nm}$ to $632.992 \mathrm{~nm}$ ).

The reference beam (of the standard laser) and the beam of the measured laser are coaxially aligned by the mirror and the splitter, thus the pulsation with beat frequency $\Delta v=\left|v_{1}-v_{2}\right|$ is produced. These beats are counted by detector with highly sensitive photodiode. The power absorbed by photo-detector is proportional to $\left(E_{1} \cos 2 \pi t v_{1}+E_{2} \cos 2 \pi t v_{2}\right)^{2}$ which is, according to trigonometric rules, the sum of detected terms $\left(E_{1}^{2}+E_{2}^{2}\right) / 2$, and $E_{1} E_{2} \cos 2 \pi t \Delta v$, and of optic-frequencies' terms that are not detected (Husein et al, 2010).

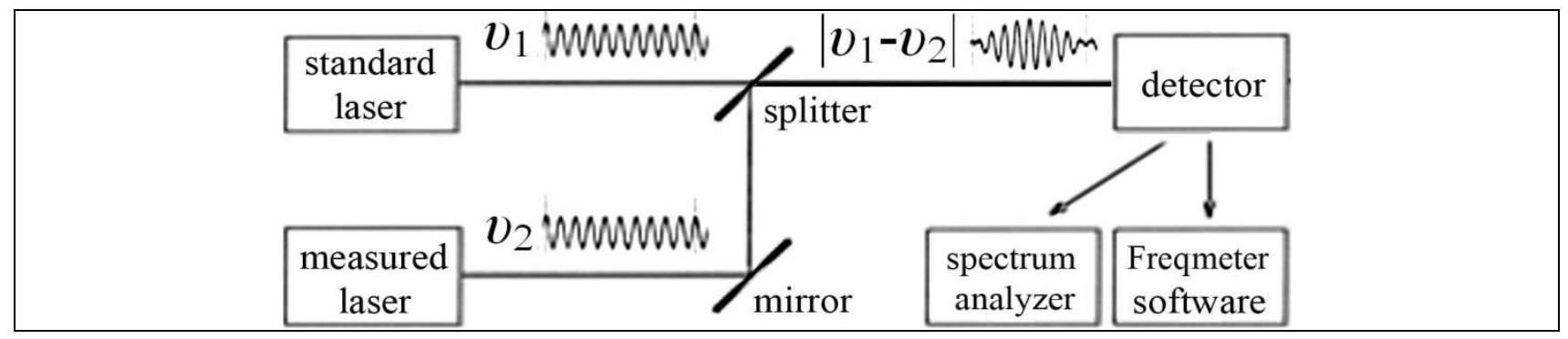

Fig. 4. Principle of beat frequency measurement 
The Freqmeter program, connected to the detector, monitors the beat frequency $\Delta v$ in the range from $1 \mathrm{MHz}$ to $1 \mathrm{GHz}$; and enables evaluation of standard deviation at different sampling periods.

Interferometer lasers usually have two frequencies, a horizontal polarized component and a vertical polarized component, that differ with a constant in the range (1.5 to 2) $\mathrm{MHz}$. The polarization directions of both lasers must be aligned, using a half-wave plate for rotation and a linear polarizer to select one of two components. Longitudinal alignment and transversal alignment of the beams can be checked by signal observation on the frequency analyzer during stable operation of the measured laser: the signal has to be symmetrical and its central frequency has to be equal to the average frequency measured by the Freqmeter program (Fig. 5.a/b). The selected peak $v_{1}$ should not be too close to the measured frequency $v_{2}$, otherwise $\Delta v$ might cross zero frequency during the measurement period owing to variation of $v_{2}$. Further details about the calibration procedure optimization have been reported (Lipus et al, 2013).
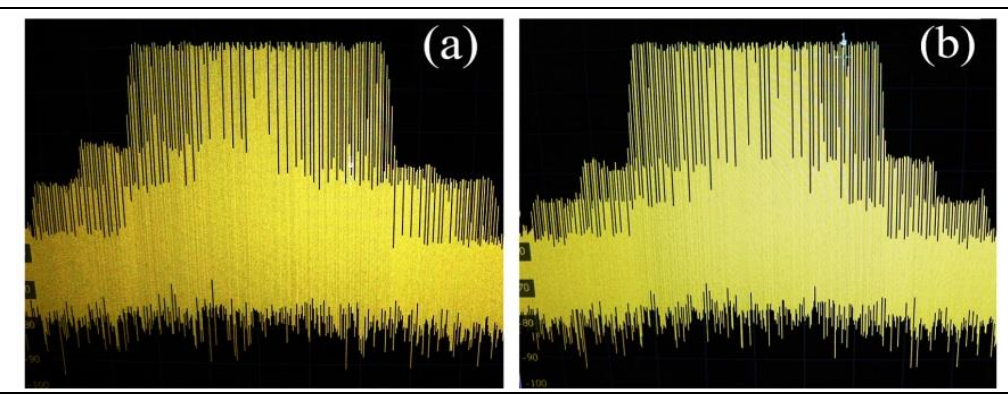

Fig. 5. Beat frequency signal of HP laser at f-peak:

(a) An asymmetrical signal before alignment of polarized components

(b) A symmetrical signal after proper alignment

\section{Verification of calibration accuracy}

The relative expanded uncertainty of MIRS1is expected by MeP to be $5 \mathrm{E}^{-11}$ (Quinn, 2003), while at CCl-K11 its uncertainty was determined even smaller, comparable to other participants (Matus et al, 2010). The expanded uncertainty of the calibration procedure for laser interferometers was certified as $0.5 \mathrm{MHz}\left(10^{-9}\right.$ relatively), i.e. 20-times higher than the frequency standard.

Regarding the use of the laser as a primary standard in the national traceability chain, the further task is assuring its accuracy level by periodically verifying the accuracy of the calibration setup. One way is inter-laboratory comparison by measuring beat frequency of two similar primary lasers. This has to be organized on the international level and demands the transportation of the laser, which is sensitive to the mechanical vibrations, and some risk exists that its proper functioning might be affected. Anyhow, it is desired to make frequent tests inside the laboratory. The method presented here is simple, quick and precise enough to confirm the expected accuracy, only a highly stable secondary laser is needed for such test, installed into the calibration setup. 
HeNe interferometer laser Hewlett Packard (HP), owned by the laboratory, was found a stable enough for short-time measurements. Firstly, the resolution of beat frequency detection of HP was examined for all seven peaks. The frequency differences among these lines range from a few tens of $\mathrm{MHz}$ up to a few hundred of $\mathrm{MHz}$ (Fig. 3d). To compare measured peak-peak differences, a stable range of HP, that appears four hours after switching it on (Fig. 6), was chosen. The beat frequency was measured at different peaks successively (10-sec sampling period, 50-times at each peak, switching from one to another peak by quick locking within 1 minute). Some results are presented in Tab. 1. The measured differences were calculated from the beat frequencies for pairs of successively locked peaks.
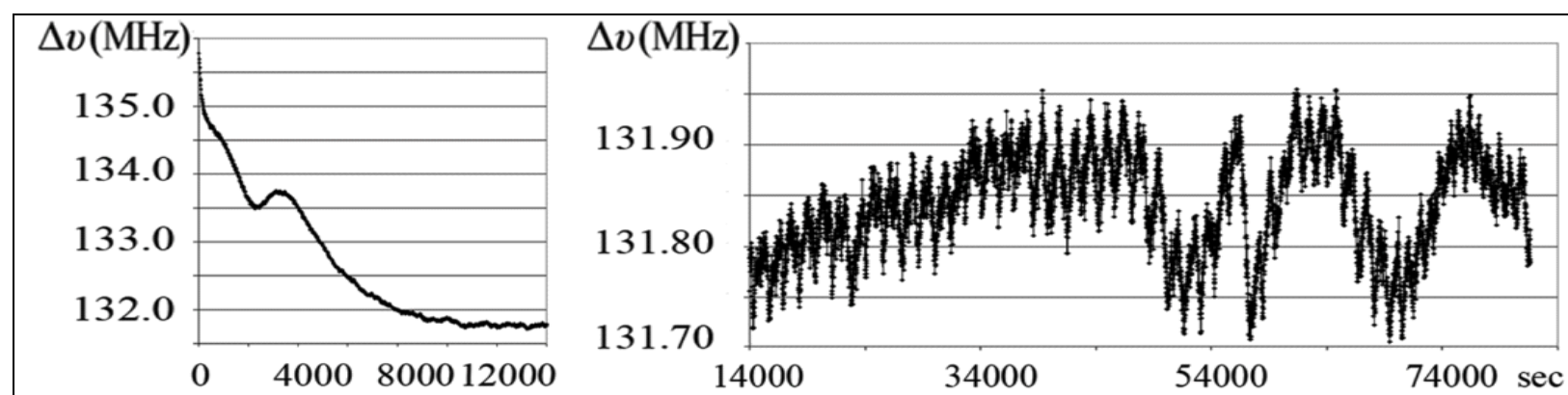

Fig. 6. Beat frequency measurement of HP laser at $\mathrm{f}$ peak (10-sec sampling).

\begin{tabular}{|l|c|c|c|c|c|c|}
\hline $\begin{array}{l}\text { Pea } \\
\mathrm{k}\end{array}$ & $\begin{array}{c}\text { Beat } \\
\text { frequency } \\
\text { (MHz) }\end{array}$ & $\begin{array}{c}\text { Standard } \\
\text { deviation } \\
\text { (MHz) }\end{array}$ & Peak-peak & $\begin{array}{c}\text { Measured } \\
\text { difference } \\
\text { (MHz) }\end{array}$ & $\begin{array}{c}\text { Theoretical } \\
\text { difference } \\
\text { (MHz) }\end{array}$ & $\begin{array}{c}\text { Deviation* } \\
\text { (MHz) }\end{array}$ \\
\hline d & 158.03 & 0.01 & & & & \\
e & 145.18 & 0.01 & d-e & 12.85 & 12.861 & $\mathbf{- 0 . 0 1}$ \\
\hline f & 131.81 & 0.015 & e-f & 13.37 & 13.363 & $\mathbf{+ 0 . 0 1}$ \\
\hline g & 118.60 & 0.01 & f-g & 13.21 & 13.198 & $\mathbf{+ 0 . 0 1}$ \\
\hline h & 14.87 & 0.015 & g-h & 103.73 & 103.755 & $\mathbf{- 0 . 0 2 5}$ \\
\hline j & 28.63 & 0.015 & j+h & 43.50 & 43.504 & $\mathbf{- 0 . 0 1}$ \\
\hline j & 28.65 & 0.01 & j-j & 0.02 & 0 & $\mathbf{+ 0 . 0 2}$ \\
\hline
\end{tabular}

Tab. 1. Deviation of peak-peak difference (*the measured minus the theoretical).

Theoretical differences are taken from the list in Fig. 3.d. Regarding the location of $\mathrm{HP}$ frequency in the range of triplet (lower than h-frequency and just a few $\mathrm{MHz}$ higher than i-frequency); the pulsation frequencies at peaks that are inside quadruplet are subtracted, while for $\mathrm{j}$, h pair are summarized. Deviations of measured difference from the theoretical are presented in the last column (Tab. 1). They are mainly attributed to HP laser's variability. The uncertainty of MIRS1 frequency is lower (Matus et al, 2010); and it would contribute variations to the $3^{\text {rd }}$ decimal place. The uncertainty of Freqmeter detection is negligible as a result of to the stability of internal oscillator in the detector lower than $10^{-6}$.

The deviations prove that the accuracy of the whole setup is better than 0.5 $\mathrm{MHz}$, the certified expanded uncertainty; so the setup is adequate for observation of $\mathrm{HeNe}$ interferometer lasers with stability as good as $10^{-9}$. Furthermore, applying such 
interferometer laser for precise dimensional measurements, its frequency has minor influence on overall interferometry accuracy due to several other influencing factors. Among environmental, geometric and instrumental errors, the most influential contributor to the uncertainty is temperature. Wavelength compensation, the material thermal compensation and the dead path correction are inevitable, but still have usually a greater contribution to the overall uncertainty than the laser itself.

\section{Conclusion}

The metrological characteristics of the applied portable digitally controlled iodine stabilized HeNe laser were investigated in the framework of The Metrology Institute of the Republic of Slovenia. By participating in CCL-K11 international comparison the laser was verified as highly accurate for all applications in metrological chains requiring the primary frequency stability. The relevant calibration and measurement capabilities are included in the CIPM database since 2010 and in the scope of accreditation since 2014. The laser is applied for calibrating of secondary HeNe lasers that are used for laboratory and industrial interferometry.

Reliable primary frequency standard is of highest national metrological interest in the sense of assuring national traceability chains to high level industrial calibrations and measurements. Therefore, periodical tests of the calibration capability are needed. The highly precise way is comb measurement at a pilot laboratory, recommended by CIPM at least once in ten years. Another way is interlaboratory comparison measuring the beat frequency of two similar primary lasers, yielding a mutual uncertainty for both lasers, recommended every few years. Both ways are organized on the international level and demand laser's transportation. For frequent tests inside the laboratory, a less precise, jet simple and effective, method is a short measurement using a highly-stable secondary laser at successively locked peaks, as presented in this contribution. The test proved that the calibration setup is adequate for observation of HeNe interferometer lasers with stability as good as $10^{-9}$, and it is included into the annual protocol for maintaining traceability of LTM.

\section{Acknowledgement}

The project was co-financed by the Metrology Institute of the Republic of Slovenia (MIRS) within the C3212-10-000072 contract on activities and obligations of the holder of the national standard of length.

\section{References}

Acko, B.; Brezovnik, S. \& Sluban, B. (2013). Verification of software applications for evaluating interlaboratory comparison results, 24th DAAAM International Symposium, B. Katalinic (Ed.), Oct. 2013, Zadar, Croatia, Vol. 24, No. 1, ISSN 23041382, DAAAM International Vienna, Vienna, Procedia Engineering 69 (2014), 263272, DOI: 10.1016/j.proeng.2014.02.231 
C. Lipus, L.; Budzyn, G.; Rzepka, J. \& Acko, B.: Calibration Capability With Laser...

Acko, B.; Sluban, B.; Tasic, T. \& Brezovnik, S. (2014). Performance metrics for testing statistical calculations in interlaboratory comparisons. Advances in Production Engineering \& Management, Vol. 9, No. 1, 44-52, ISSN 1854-6250

Acko, B.; Brezovnik, S.; Lipus, L.C. \& Klobucar, R. (2015). Verification of statistical calculations in interlaboratory comparisons by simulating input datasets. International Journal of Simulation Modelling, Vol. 14, No. 2, 227-237, ISSN 17264529, DOI: 10.2507/IJSIMM14(2)4.288

Budzyn, G.; Dudzik, G. \& Rzepka, J. (2007). Portable digitally controlled iodine stabilized He-Ne laser with very long lock-up time. Conference Information: 2007 ICTON Mediterranean Winter Conference, pp. 167-169, ISBN 978-1-4244-1639-4, Sousse, Dec. 2007, Tunisia, DOI: 10.1109/ICTONMW.2007.4446975

Budzyn, G. \& Rzepka, J. (2008). Back-reflection effects in a frequency-stabilized two mode He-Ne laser. Optics Communications, Vol. 281, No. 22, 5592-5595, ISSN 0030-4018, DOI: 10.1016/j.optcom.2008.07.082

Godina, A. \& Acko, B. (2012). Calibration of tape measures with small measurement uncertainty, DAAAM International Scientific Book 2012, pp. 187-196, B. Katalinic (Ed.), Published by DAAAM International, ISBN 978-3-901509-86-5, ISSN 17269687, Vienna, Austria, 4244-1639-4, Sousse, December 2007, Tunisia, DOI: 10.2507/daaam.scibook.2012.16

Vorotnikov, A.; Bashevskaya, O.; Ilyukhin, Y.; Romash, E.; Isaev, A.V. \& Poduraev, Y. (2016). Geometrical approach for industrial robot axis calibration using laser tracker, Proceedings of the 26th DAAAM International Symposium, pp.0897-0904, B. Katalinic (Ed.), Pub. by DAAAM International, ISBN 978-3-902734-07-5, ISSN 1726-9679, Vienna, Austria,

Husein, H.; Sobee, M.A.; Amer, M. (2010). Calibration of Michelson-type laser wavemeter and evaluation of its accuracy, Optics and Lasers in Engineering, Vol. 48, No. 3, 393-397, ISSN 0143-8166,

Lipus, L.C.; Matus, M. \& Acko, B. (2013). Optimization of calibrating HeNe laser interferometers by sample-period simulation. International Journal of Simulation Modelling, Vol. 12, No. 3, 154-163, ISSN 1726-4529,

Matus, M.; Nyholm, K.; Madej, A.; Bernard, J.E.; Walczuk, J.; Lipus, L.C. et al. (2010). The CCL-K11 ongoing key comparison 2007 - 2009, Metrologia, Vol. 47, Supp. 1, ISSN 0026-1394, DOI: 10.1088/0026-1394/47/1 A/04009

Mudronja, V.; Katic, M. \& Simunovic, V. (2014). Realization of the highest level of traceability in Croatian National Laboratory for Length. Transactions of Famena, Vol. 38, No. 1, 37-44, ISSN 1333-1124

Quinn, T.J. (2003). Practical realization of the definition of the metre, including recommended radiations of other optical frequency standards (2001). Metrologia, Vol. 40, No. 2, 103-133, ISSN 0026-1394, DOI: 10.1088/0026-1394/40/2/316 Reihle, F. (2004). Frequency standards, WILEY-VCH Verlag, ISBN 3-527-40230-6, Germany 